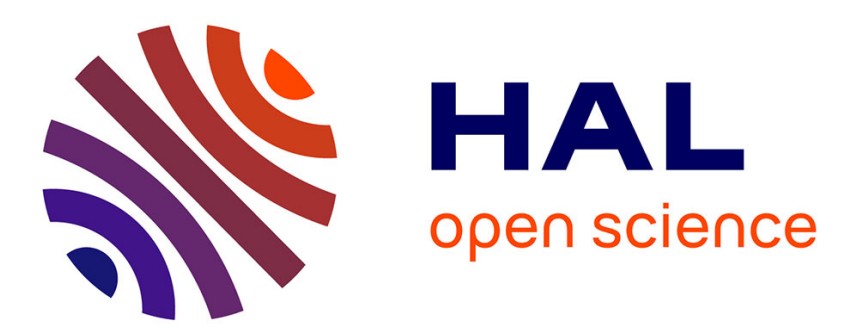

\title{
Effet de la dilution de suspensions de kaolinite et de montmorillonite sur l'adsorption de l'atrazine
}

\author{
Martine Terce, Claude Picot
}

\section{To cite this version:}

Martine Terce, Claude Picot. Effet de la dilution de suspensions de kaolinite et de montmorillonite sur l'adsorption de l'atrazine. Agronomie, 1983, 3 (9), pp.883-890. hal-00884585

\section{HAL Id: hal-00884585 \\ https://hal.science/hal-00884585}

Submitted on 1 Jan 1983

HAL is a multi-disciplinary open access archive for the deposit and dissemination of scientific research documents, whether they are published or not. The documents may come from teaching and research institutions in France or abroad, or from public or private research centers.
L'archive ouverte pluridisciplinaire HAL, est destinée au dépôt et à la diffusion de documents scientifiques de niveau recherche, publiés ou non, émanant des établissements d'enseignement et de recherche français ou étrangers, des laboratoires publics ou privés. 


\title{
Effet de la dilution de suspensions de kaolinite et de montmorillonite sur l'adsorption de l'atrazine
}

\author{
Martine TERCE \\ avec la collaboration technique de Claude PICOT \\ I.N.R.A., Station de Science du Sol, Route de Saint-Cyr F 78000 Versailles
}

RÉSUMÉ

\begin{abstract}
L'étude de l'adsorption d'une base faible organique, l'atrazine $\left(\mathrm{pK}_{\mathrm{BH}}+=1,68\right)$, par une kaolinite et une montmorillonite montre que les isothermes d'adsorption sont différentes selon les dilutions des suspensions d'argile quel que soit le cation compensateur ( $\mathrm{Na}, \mathrm{Ca}$ ou $\mathrm{Al}$ ). Les quantités fixées, rapportées au gramme de substrat, pour une même concentration de la solution à l'équilibre, sont d'autant plus grandes que la masse d'adsorbant est plus petite. Des mesures potentiométriques montrent que le $\mathrm{pH}$ des solutions avant et après contact avec les argiles est le seul paramètre qui change pendant la mesure. Dans le cas de la kaolinite, il semble que ce sont les charges variables dues à la dissociation des groupements hydroxylés des surfaces latérales des argiles qui sont responsables de ce phénomène de dilution. L'effet du cation compensateur sur l'adsorption incite à dire que le principal mécanisme de fixation de l'atrazine est une protonation de la molécule au voisinage de la surface, suivie d'une interaction coulombienne entre le cation organique et la charge négative variable de la kaolinite. Par analogie, ce mécanisme d'adsorption est étendu à la montmorillonite.
\end{abstract}

Mots clés additionnels : Argile, base faible organique, charge variable de surface, protonation, acidité de surface.

Additional key words : Clay, weak organic base, variable charge of surface, protonation, surface acidity.

\section{INTRODUCTION}

L'atrazine (2 chloro-4 éthylamino-6 isopropylamino-striazine) appartient à la famille des s-triazines, molécules organiques peu solubles dans l'eau, à caractère basique faible et à propriété herbicide.

L'étude de l'adsorption de l'atrazine par les constituants argileux, et principalement par la montmorillonite, a déjà fait l'objet de nombreuses études, mais il est difficile de généraliser les résultats publiés et de dégager les mécanismes responsables de la fixation, principalement dans des milieux voisins de la neutralité. Le tableau 1 illustre bien la diversité des résultats sur l'adsorption de l'atrazine par des montmorillonites du Wyoming de type Volclay, saturées par du sodium. Les quantités fixées sont comparées à partir des isothermes d'adsorption, à $20^{\circ} \mathrm{C}$, pour une même concentration d'atrazine en solution à l'équilibre. Il est possible que ces montmorillonites, pourtant de même provenance, ne soient pas strictement identiques, mais une variation de 1 à 50 et même davantage, dans les quantités d'atrazine fixées, ne s'explique paś uniquement par de très légères différences dans la composition du minéral. Cependant, il faut noter que l'adsorption est d'autant plus importante que la concentration en argile dans la suspension est plus faible.

BAR-ON \& SHAINBERG (1970) et FrenkEl \& SuAREZ (1977) montrent qu'une augmentation de la dilution de la suspension de montmorillonite entraîne une augmentation de l'hydrolyse du minéral, même à des $\mathrm{pH}$ voisins de la 
TABLEAU 1

Comparaison des quantités d'atrazine fuxées par des montmorillonites sodiques, du Wyoming (Volclay) pour une même concentration dans la solution à l'équilibre $\left(C_{e q}=45 \times 10^{-3} \mu \mathrm{M} / \mathrm{ml}\right)$, à $20^{\circ} \mathrm{C}$.

Comparison of the quantity of atrazine adsorbed by sodic Wyoming montmorillonites (Volclay), at the same equilibrium concentration in solution $\left(C_{e q}=45 \times 10^{-3} \mu\right.$ mole $\left./ \mathrm{ml}\right)$ at $20^{\circ} \mathrm{C}$.

\begin{tabular}{lccc}
\hline \multicolumn{1}{c}{ Auteurs } & $\mathrm{pH}$ de la suspension & Quantités fixées $(\mu \mathrm{M} / \mathrm{g})$ & $\begin{array}{c}\text { Concentration de l'argile } \\
\text { dans la suspension } \\
\text { lors de la mesure d'adsorption }\end{array}$ \\
\hline WEBER (1970) & 6,8 & 50 & $0,625 \mathrm{~g} / 1$ \\
\hline BALLEY et al. $(1958)$ & 6,5 & 1 & $10 \mathrm{~g} / 1$ \\
\hline VALLET et al. $(1973)$ & 6,9 & 0,2 & $25 \mathrm{~g} / 1$ \\
\hline \hline
\end{tabular}

neutralité. Or, les travaux de TERCE \& CALVET $(1974,1975$, 1977) indiquent que l'apparition d'aluminium à la surface de l'argile augmente la capacité d'adsorption du minéral vis-àvis de l'atrazine. On peut donc penser que l'hydrolyse du réseau de l'argile, entraînant l'apparition d'aluminium à la surface, est principalement responsable des différences dans les quantités fixées (tabl. 1).

L'objectif de cet article est d'interpréter cet effet de dilution de la suspension sur les quantités d'atrazine adsorbée et de voir le rôle de l'aluminisation du minéral dans ce phénomène.

Les argiles utilisées pour cette étude sont, d'une part, une montmorillonite et, d'autre part, une kaolinite, aussi sensible à l'hydrolyse qu'une montmorillonite, mais pour laquelle les phénomènes d'adsorption et d'hydrolyse se limitent aux surfaces externes.

\section{MATÉRIEL ET MÉTHODES}

La kaolinite originaire de Saint-Austell (Grande-Bretagne) a une capacité d'échange cationique de $1,2 \mathrm{meq} / 100 \mathrm{~g}$ à $\mathrm{pH} 7$ et une surface spécifique de $14 \mathrm{~m}^{2} / \mathrm{g}$. Elle est saturée naturellement par du sodium et du calcium. Elle est utilisée soit dans son état naturel, soit saturée par du sodium, du calcium ou de l'aluminium.

La montmorillonite est une bentonite Volclay du Wyoming. Sa capacité d'échange cationique est de $94 \mathrm{meq} / 100 \mathrm{~g}$ et son aire de surface externe plus interne est de l'ordre de $800 \mathrm{~m}^{2} / \mathrm{g}$. Elle est saturée par du sodium, du calcium ou de l'aluminium (les cations sont apportés sous forme de chlorure et l'argile est ensuite lavée jusqu'à la disparition des ions $\mathrm{Cl}^{-}$dans les eaux de lavage).

L'atrazine, de formule chimique :<smiles>CCCCCCCCCNc1nc(Cl)nc(N[TlH])n1</smiles>

est une molécule à caractère acido-basiquc

$$
\left(\mathrm{pK}_{\mathrm{BH}}+=1,68\right) \text {. }
$$

Sa solubilité dans l'cau est de $30 \mathrm{mg} / \mathrm{l}$ et son poids molé. culaire de $215 \mathrm{~g} / \mathrm{M}$.
Les molécules d'atrazine utilisées sont fournies par CibaGeigy. Les solutions de base sont constituées d'un mélange de molécules marquées au ${ }^{14} \mathrm{C}$ sur le noyau aromatique (activité spécifique de $24,9 \mu \mathrm{Ci} / \mathrm{mg}$ et pureté radiochimique supérieure a 99 p. 100) et de molécules non marquées (pureté chimique de 99,8 p. 100).

\section{A. Détermination des isothermes d'adsorption}

Les isothermes d'adsorption sont obtenues dans les conditions suivantes :

- Utilisation de molécules d'atrazine marquées au ${ }^{14} \mathrm{C}$.

- Agitation à $20^{\circ} \mathrm{C}$, d'une masse de $\mathrm{m}$ grammes d'argile, avec $\mathrm{V} \mathrm{ml}$ de solution d'atrazine de concentration initiale variable $\mathrm{Ci}$ (en $\mathrm{mg} / \mathrm{l})$ au $\mathrm{pH}$ de l'eau bipermutée $(\mathrm{pH}$ de l'ordre de 6).

- Temps de contact de $48 \mathrm{~h}$, nécessaire à l'équilibre d'adsorption et mesuré préalablement.

- Centrifugation des suspensions à $40000 \mathrm{~g}$ et à $20^{\circ} \mathrm{C}$, détermination de la concentration à l'équilibre $\mathrm{Ce}$ (en $\mathrm{mg} / \mathrm{l}$ ) de la solution surnageante. Les radioactivités des solutions initiales et à l'équilibre sont déterminées à l'aide d'un compteur à scintillation liquide intertechnique SL 4000.

Les quantités fixées $Q(e n \mu g / g)$ sont calculées par la méthode des restes et sont égales à : $\mathrm{Q}=\frac{(\mathrm{Ci}-\mathrm{Ce})}{\mathrm{m}} \mathrm{V}$.

\section{B. Effet de l'aluminisation du réseau des argiles sur l'adsorption}

L'influence de l'aluminisation du réseau de l'argile sur l'adsorption est appréciée en étudiant l'effet du prétraitement de l'argile dans l'eau sur les quantités d'atrazine fixée. L'expérience est conduite de la façon suivante :

- Agitation à $20^{\circ} \mathrm{C}$ de masses variables d'argiles (kaolinite naturelle et montmorillonite $\mathrm{Na}$ ) avec $10 \mathrm{ml}$ d'eau pendant un temps de contact variable, compris entre 1 et $8 \mathrm{j}$.

- Centrifugation et retrait des surnageants.

- Addition de volumes différents de solution d'atrazine à $25 \mathrm{mg} / 1$ pour avoir, lors de la mesure d'adsorption, le même rapport adsorbant/solution, quelle que soit la masse d'argile initiale.

\section{Mesures potentiométriques des solutions aqueuses au contact des argiles}

Les $\mathrm{pH}$ des solutions aqueuses sont mesurés avant et après contact avec la kaolinite naturelle ou sodique et la 
montmorillonite $\mathrm{Na}$, pour différentes concentrations d'argile en suspension. Pour les mesures avec la kaolinite naturelle sèche, le $\mathrm{pH}$ initial est celui de l'eau bipermutée (préalablement dégazée par ébullition), soit un $\mathrm{pH}$ de 6,6. Pour la kaolinite et la montmorillonite homioniques utilisées en suspension, le $\mathrm{pH}$ initial est obtenu en mélangeant les volumes nécessaires d'eau et de solution surnageante des suspensions d'argile, ceci afin de tenir compte de la composition ionique des solutions se trouvant préalablement au contact des cristallites. Le pH final est mesuré après $48 \mathrm{~h}$ de contact argile-eau dans les conditions de la mesure d'adsorption.

\section{RESULTATS EXPERIMENTAUX}

\section{A. Isothermes d'adsorption}

La figure 1 représente les isothermes d'adsorption de l'atrazine, par la kaolinite naturelle, pour différentes

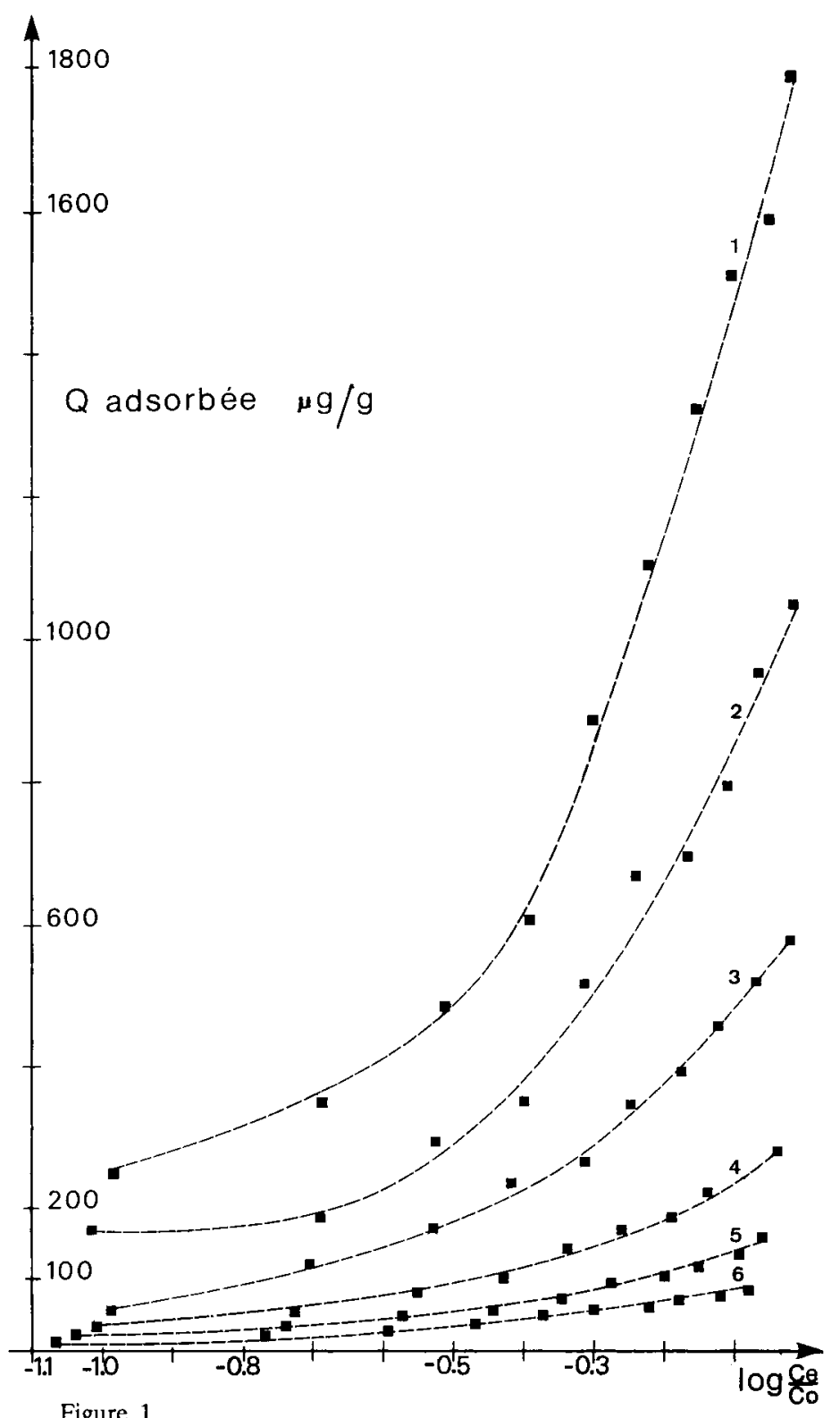

Figure 1

Influence de la dilution des suspensions de kaolinite naturelle sur les isothermes d'adsorption de l'atrazine. Les concentrations des suspensions utilisées sont: (1) $2,5 \mathrm{~g} / \mathrm{l}$, (2) $5 \mathrm{~g} / \mathrm{l}$, (3) $10 \mathrm{~g} / \mathrm{l}$, (4) $25 \mathrm{~g} / \mathrm{l}$, (5) $50 \mathrm{~g} / \mathrm{l}$, (6) $100 \mathrm{~g} / \mathrm{l}$.

Influence of natural kaolinite dilution on the atrazine adsorption isotherms. Concentrations of suspensions used: (1) $2,5 \mathrm{~g} / \mathrm{l}$, (2) $5 \mathrm{~g} / \mathrm{l}$, (3) $10 \mathrm{~g} / \mathrm{l}$, (4) $25 \mathrm{~g} / \mathrm{l}$, (5) $50 \mathrm{~g} / \mathrm{l}$, (6) $100 \mathrm{~g} / \mathrm{l}$. concentrations d'argile en suspension : $100 \mathrm{~g} / 1,50 \mathrm{~g} / 1,25 \mathrm{~g} / \mathrm{l}$, $10 \mathrm{~g} / 1,5 \mathrm{~g} / 1$ et $2,5 \mathrm{~g} / 1$. On a porté sur cette figure les quantités adsorbées exprimées en $\mu \mathrm{g} / \mathrm{g}$ d'adsorbant en fonction du log de $\mathrm{Ce} / \mathrm{Co}$, Co étant la concentration de la solution saturée $(\mathrm{Co}=30 \mathrm{mg} / \mathrm{l})$ et $\mathrm{Ce}$ la concentration de la solution à l'équilibre. Ce mode de représentation a été utilisé par CASES $(1968,1979)$ pour décrire l'adsorption de tensio-actifs. Soit $\mu_{\mathrm{s}}$ le potentiel chimique de la molécule dans la solution et soit $\mu_{C_{0}}$ le potentiel chimique de la molécule dans la solution saturée :

$\mu_{\mathrm{s}}=\mu_{\mathrm{o}}+\mathrm{RT} \ln \mathrm{a}_{\mathrm{s}}$

$\mu \mathrm{C}_{\mathrm{o}}=\mu_{\mathrm{o}}+\mathrm{RT} \ln \mathrm{a}_{\mathrm{Co}}$

En solution diluée, $\Delta \mu=\mu_{s}-\mu_{C o}=R T \ln \frac{C_{S}}{C_{0}}$ est à l'équilibre d'adsorption, $\Delta \mu=\mathrm{RT} \ln \frac{\mathrm{Ce}_{\mathrm{e}}}{\mathrm{C}_{\mathrm{o}}}$.

Cette représentation a l'avantage de relier les quantités d'atrazine adsorbées aux variations du potentiel chimique de la molécule organique dans la solution à l'équilibre avec la phase adsorbée, l'état de référence du soluté étant la solution saturée.

La figure 1 montre que les isothermes d'adsorption sont différentes selon les dilutions de l'adsorbant et que la quantité d'atrazine fixée, rapportée au gramme d'adsorbant, est d'autant plus grande que la masse de kaolinite est plus faible.

La figure $2 \mathrm{a}$ indique que le même phénomène apparaît lorsque la kaolinite est saturée soit par du sodium, soit par du calcium, soit par de l'aluminium. L'effet de dilution sur les quantités d'atrazine fixées existe également avec la montmorillonite sodique, calcique ou alumineuse (fig. 2b). L'effet du cation échangeable sur l'adsorption est illustré par la figure 3, pour une concentration en argile de l'ordre de 2,5 g/l. Pour la kaolinite (fig. 3a), l'adsorption varie dans le sens kaolinite $\mathrm{Na}>$ kaolinite $\mathrm{Ca}>$ kaolinite $\mathrm{Al}$, alors que pour la montmorillonite (fig. $3 \mathrm{~b}$ ) l'adsorption varie dans le sens montmorillonite $\mathrm{Al}>$ montmorillonite $\mathrm{Na}>$ montmorillonite $\mathrm{Ca}$. La comparaison des isothermes d'adsorption de l'atrazine par la kaolinite et la montmorillonite montre qu'avec le sodium ou le calcium, la fixation n'est pas très différente entre les 2 argiles, pour une même dilution. Par contre, l'adsorption est plus importante par la montmorillonite Al que par la kaolinite Al.

\section{B. Influence de l'aluminisation du réseau des argiles sur l'adsorption}

L'aluminisation du réseau de la montmorillonite $\mathrm{Na}$ ou $\mathrm{Ca}$ en milieu neutre est plus importante quand la concentration de l'argile dans la suspension aqueuse est faible et quand le temps de contact argile-eau est important (BAR-ON \& ShainberG, 1970 ; Frenkel \& SuAReZ, 1977).

Si cette aluminisation du réseau est le phénomène responsable de l'adsorption d'atrazine, on doit observer un accroissement des propriétés adsorbantes de l'argile à la suite d'un prétraitement par l'eau, l'effet de ce prétraitement doit être d'autant plus important que le temps de contact argile-eau est plus long ou que la dilution de l'argile est plus grande. Les résultats expérimentaux présentés sur la figure 4 montrent que ce n'est pas le cas : lorsqu'à la suite du prétraitement, les mesures d'adsorption sont faites dans le même rapport adsorbant/solution, les quantités d'atrazine fixées sont identiques quels que soient le temps de contact et la dilution de l'argile lors du prétraitement. 


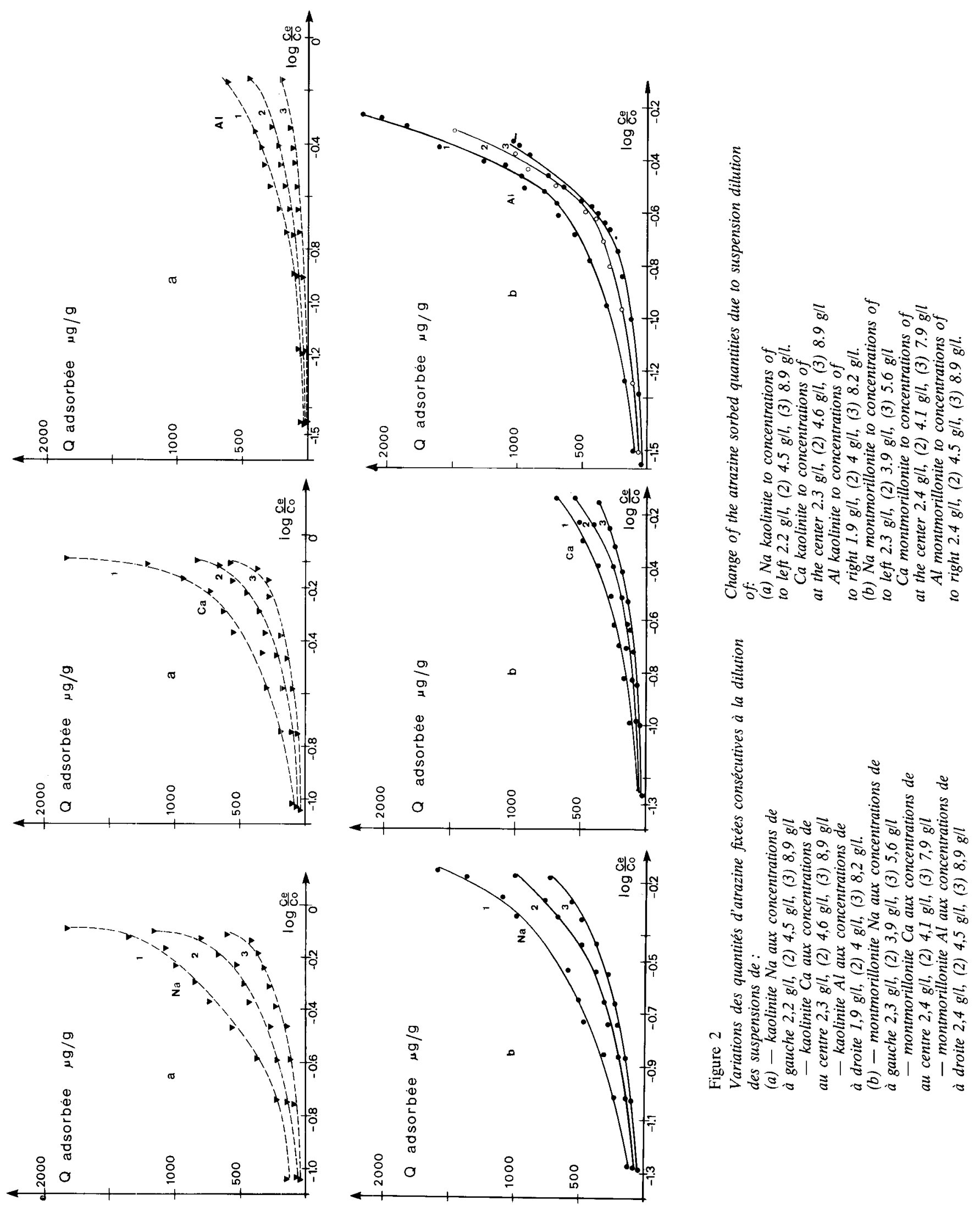



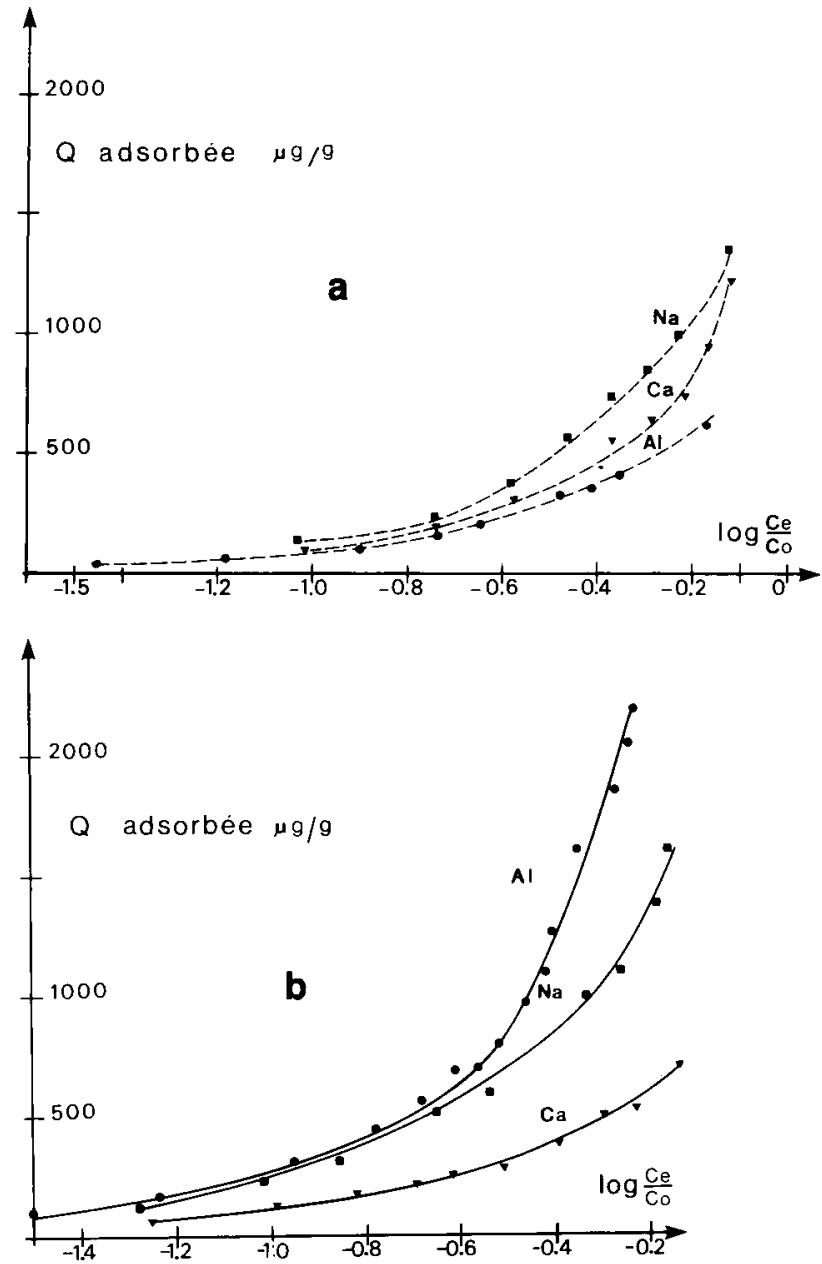

Figure 3

Effet du cation saturant l'argile sur l'adsorption de l'atrazine par (a) - la kaolinite (dilution des suspensions de l'ordre de $2,5 \mathrm{~g} / \mathrm{l}$ ) (b) - la montmorillonite (dilution des suspensions de l'ordre de $2,5 \mathrm{~g} / \mathrm{l})$.

Effect of the clay compensating cation on atrazine adsorption by: (a) kaolinite (suspensions dilution in the order of $2.5 \mathrm{~g} / \mathrm{l}$ )

(b) montmorillonite (suspensions dilution in the order of $2.5 \mathrm{~g} / \mathrm{l}$ ).

\section{Mesures potentiométriques}

Les valeurs de $\mathrm{pH}$ mesurées avant et après contact avec les argiles figurent dans les tableaux 2 et 3 . Le tableau 2 montre que le $\mathrm{pH}$ final de la solution varie selon les dilutions de la kaolinite. On note aussi bien pour la kaolinite

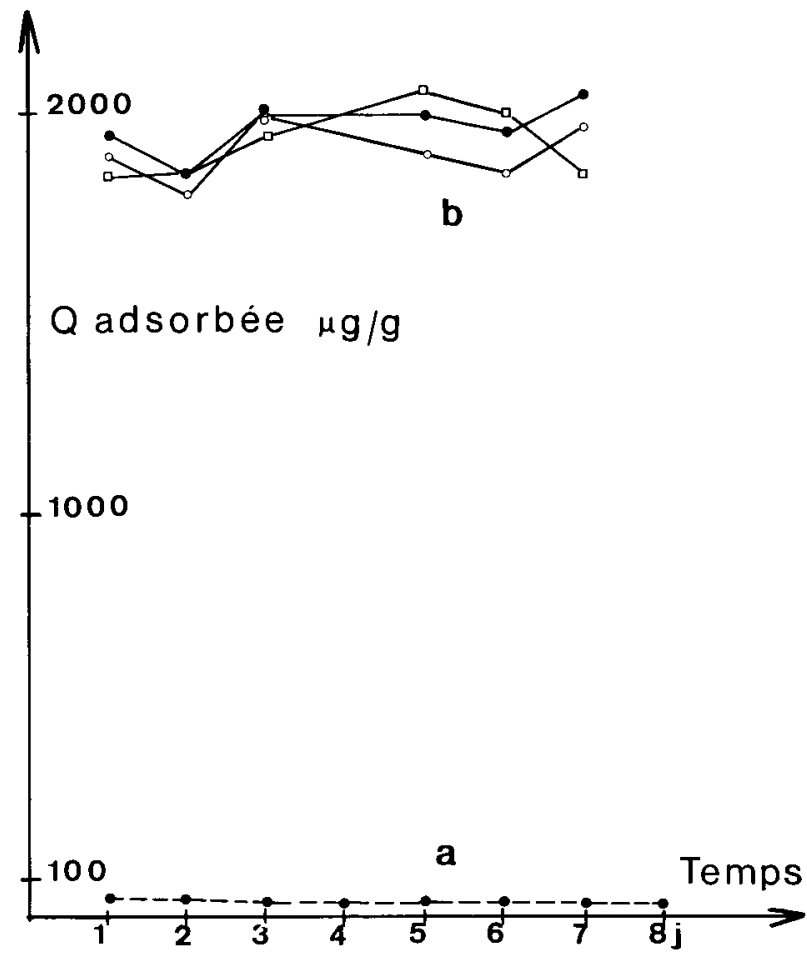

Figure 4

Influence du prétraitement de l'argile dans l'eau sur la mesure d'adsorption d'atrazine, pour différentes dilutions de:

(a) - kaolinite naturelle aux concentrations de $20 \mathrm{~g} / \mathrm{l}$, $50 \mathrm{~g} / \mathrm{l}$, - $100 \mathrm{~g} / \mathrm{l}$

Les mesures d'adsorption sont effectuées sur des suspensions de $100 \mathrm{~g} / \mathrm{l}$. La concentration à l'équilibre est de $19,6 \mathrm{mg} / \mathrm{l}$.

(b) - montmorillonite $\mathrm{Na}$ aux concentrations de $\square 2,5 \mathrm{~g} / \mathrm{l}, \bigcirc 5 \mathrm{~g} / \mathrm{l}$, - $10 \mathrm{~g} / \mathrm{l}$.

Les mesures d'adsorption sont effectuées sur des suspensions de $2 \mathrm{~g} / \mathrm{l}$. La concentration à l'équilibre est de $21,1 \pm 0,3 \mathrm{mg} / 1$.

Effect of clay pretreatment in water on the atrazine adsorption measurement, for different dilutions of:

(a) Natural kaolinite to concentrations of $-20 \mathrm{~g} / \mathrm{l}$, $-50 \mathrm{~g} / \mathrm{l}$, - $100 \mathrm{~g} / \mathrm{l}$

Adsorption measurements on suspensions of $100 \mathrm{~g} / \mathrm{l}$.

Equilibrium concentration $19,6 \mathrm{mg} / \mathrm{l}$.

(b) - Na montmorillonite to concentrations of $\square 2.5 \mathrm{~g} / \mathrm{l}, \bigcirc 5 \mathrm{~g} / \mathrm{l}$, - $10 \mathrm{~g} / \mathrm{l}$. Adsorption measurements on suspensions of $2 \mathrm{~g} / \mathrm{l}$. Equilibrium concentration $21.1 \pm 0.3 \mathrm{mg} / \mathrm{l}$.

naturelle que pour la kaolinite sodique, une diminution de pH consécutive à l'apport d'argile en suspension. Rapportée au gramme d'adsorbant, la libération de protons est d'autant plus importante que la concentration en argile est plus faible. Cependant, à dilution égale, la libération de

TABLEAU 2

Influence de la dilution de la kaolinite, soit naturelle, soit sodique, dans une suspension aqueuse sur la libération de protons dans la solution. Influence of natural or sodic kaolinite dilution in an aqueous suspension on proton release into solution.

\begin{tabular}{|c|c|c|c|c|c|}
\hline & $\mathrm{pH}$ initial & $\begin{array}{l}\text { Concentration } \\
\text { des suspensions } \\
\text { de kaolinite }\end{array}$ & $\begin{array}{l}\mathrm{pH} \text { final } \\
\text { de la } \\
\text { solution }\end{array}$ & $\begin{array}{c}{\left[\mathrm{H}^{+}\right] \text {libérée }} \\
\text { dans la } \\
\text { solution }\end{array}$ & $\begin{array}{c}\text { libćration } \\
\text { de }\left[\mathrm{H}^{+}\right] / \mathrm{g} \\
\text { de kaolinite }\end{array}$ \\
\hline Kaolinite naturelle & $6,60 \pm 0,01$ & $\begin{array}{rl}2,5 & \mathrm{~g} / 1 \\
5 & \mathrm{~g} / 1 \\
10 & \mathrm{~g} / 1 \\
25 & \mathrm{~g} / 1 \\
50 & \mathrm{~g} / 1 \\
100 & \mathrm{~g} / 1\end{array}$ & $\begin{array}{l}6,05 \pm 0,01 \\
5,90 \pm 0,01 \\
5,71 \pm 0,01 \\
5,47 \pm 0,01 \\
5,24 \pm 0,01 \\
5,07 \pm 0,01\end{array}$ & $\begin{array}{r}6,20 \times 10^{-7} \\
10,1 \times 10^{-7} \\
17,0 \times 10^{-7} \\
31,4 \times 10^{-7} \\
55,0 \times 10^{-7} \\
83,0 \times 10^{-7}\end{array}$ & $\begin{array}{r}248 \times 10^{-9} \\
201 \times 10^{-9} \\
170 \times 10^{-9} \\
125 \times 10^{-9} \\
110 \times 10^{-9} \\
83 \times 10^{-9}\end{array}$ \\
\hline Kaolinite sodique & $6,90 \pm 0,01$ & $\begin{array}{rl}7,3 & \mathrm{~g} / 1 \\
14,7 & \mathrm{~g} / 1 \\
29,4 \mathrm{~g} / 1\end{array}$ & $\begin{array}{l}6,52 \pm 0,01 \\
6,65 \pm 0,01 \\
6,76 \pm 0,01\end{array}$ & $\begin{array}{l}3,02 \times 10^{-7} \\
2,24 \times 10^{-7} \\
1,74 \times 10^{-7}\end{array}$ & $\begin{array}{r}41,4 \times 10^{-9} \\
15,2 \times 10^{-9} \\
5,9 \times 10^{-9}\end{array}$ \\
\hline
\end{tabular}


protons est plus importante avec la kaolinite naturelle. Ceci peut s'expliquer par le fait que la kaolinite naturelle est rapportée sous forme de poudre alors que la kaolinite sodique a subi plusieurs contacts avec des solutions salines et est amenée sous forme de suspension, aussi le milieu d'équilibre des cristallites de kaolinite est complètement différent. Le tableau 3 montre également une variation du $\mathrm{pH}$ final du surnageant de la montmorillonite Na suivant les trois dilutions : dans ce cas, le pH final augmente d'autant plus que la concentration en argile est grande, mais rapportée au gramme d'adsorbant, la fixation de protons est d'autant plus grande que la concentration en argile est faible. De mêtne que pour la kaolinite sodique, les particules de montmorillonite sont apportées sous forme de suspension et elles ont subi plusieurs contacts avec des solutions salines. Là encore, le milicu d'équilibre de la montmorillonite n'est pas forcément identique à celui de la kaolinite $\mathrm{Na}$.

\section{DISCUSSION}

L'atrazine est une base très faible $\left(\mathrm{pK}_{\mathrm{BH}}+=1,68\right)$ et les mesures d'adsorption sont faites dans des conditions de $\mathrm{pH}$ telles que les rnolécules d'atrazine: se trouvent en solution uniquement sous forme neutre. L'équilibre d'adsorption :

Atrazine (solution) $+[$ Argile $] \rightleftarrows$ Atrazine (adsorbée) [Argile]

est caractérisé par une constante d'équilibre $\mathrm{K}_{\mathrm{e}}$. Selon le principe de la loi d'action de masse, quelle que soit la masse d'adsorbant amenée au moment de la détermination de l'isotherme d'adsorption, on devrait obtenir une seule et unique isotherme, tous les autres paramètres de la mesure restant constants. Or, on obtient un faisceau d'isothermes et non une seule isotherme, quelle que soit l'argile ou le cation saturant l'argile.

Plusieurs hypothèses peuvent être posées pour expliquer ceci :

- L'état d'agrégation des particules d'argile serait différent selon les différentes concentrations des suspensions et, dans ce cas, plus les argiles sont diluées, plus les surfaces sont accessibles aux molécules d'atrazine. S'il n'existe pas à ma connaissance de travaux démontrant que dans des suspensions diluées, allant de $2,5 \mathrm{~g} / \mathrm{l}$ à $100 \mathrm{~g} / 1$, l'état d'agrégation des particules de kaolinite est variable ou non, le travail de PONS (1980) montre cependant que les cristallites de montmorillonite du Wyoming $\mathrm{Na}$ sont entièrement dispersées pour des concentrations en argile en suspension inférieures à $180 \mathrm{~g} / \mathrm{l}$. Les concentrations de montmorillonite $\mathrm{Na}$ utilisées pour les mesures d'adsorption étant bien plus petites et l'effet observé restant d'autant plus important que la dilution de l'argile est plus grande, on peut à priori estimer que ce n'est pas une différence d'état d'agrégation qui est responsable de cet effet.

- Si l'aluminisation du réseau des argiles est responsable de l'adsorption d'atrazine, on peut penser que les états différents d'aluminisation acquis lors des mesures dans différentes conclitions de dilution des suspensions sont à l'origine de l'obtention d'un faisceau d'isothermes. Les expériences avec prétraitement démontrent toutefois clairement que cette modification de l'argile n'est pas responsable de l'adsorption et que ce sont les conditions de dilution au moment de la mesure qui définissent les quantités d'atrazine fixées. Ceci exclut d'ailleurs l'hypothèse émise en introduction sur le mécanisme de l'adsorption.

- La variation du $\mathrm{pH}$ final des solutions, consécutive à l'apport différent de masse d'adsorbant dans le même volume de solution est un paramètre à prendre en compte. Si l'on examine le sens de cette variation, on s'aperçoit qu'elle n'est pas la même pour les 2 argiles. Aussi va-t-on discuter séparément de l'adsorption de l'atrazine par la kaolinite et par la montmorillonite.

\section{A. Adsorption de l'atrazine par la kaolinite}

Les travaux de SCHOFIELD \& SAMSON (1953), EKKA \& FRIPIAT (1957), FRIPIAT (1957), VESTIER (1969), CASES et al. (1971) montrent que la capacité d'échange cationique de la kaolinite, donc que la charge négative en surface, varie en fonction de l'acidité des suspensions. La charge négative est d'autant plus grande que le $\mathrm{pH}$ est plus alcalin. Cette variation de charge est due à la présence de groupements hydroxyles superficiels situés sur les surfaces latérales des particules pouvant être dissociées suivant les conditions de pH. D'après FRIPIAT (1957), c'est la présence de chaînons actifs du type $\mathrm{OH}-\mathrm{Si}-\mathrm{O}^{\left(\mathrm{H}^{+}\right)}-\mathrm{Al}-{ }^{1 / 2}<\mathrm{OH}$ qui est responsable de la charge variable. Le caractère acide des hydroxyles superficicls diffère suivant les sites et l'acidité de ces sites varic dans le sens $\mathrm{Al}^{(-)}>\mathrm{Si} \mathrm{OH}>\mathrm{Al} \mathrm{OH}>\mathrm{Si}-\mathrm{O}-\mathrm{Al}^{\left(\mathrm{H}^{+}\right)}$. Le point de charge nulle due aux groupements hydroxylés acidoïdes de la kaolinite est situé à des valeurs de $\mathrm{pH}$ très bas, de l'ordre de 2 ou 3 (VESTIER, 1969 ; CASES et al., 1971). L'immersion dans l'eau des particules de kaolinite entraîne une diminution du $\mathrm{pH}$ de l'eau (tableau 2). Cet abaissement du $\mathrm{pH}$ est attribué à la dissociation de groupements $\mathrm{OH}$ avec pour conséquence la libération de protons dans la solution et une augmentation de la charge négative globale de la surface. La libération de protons, rapportée au gramme de kaolinite, étant d'autant plus grande que l'argile est plus diluée, la densité de charge négative doit être d'autant plus importante que la masse d'argile est plus faible. Or, la fixation de l'atrazine, rapportée au gramme d'adsorbant augmente avec la dilution de la kaolinite (fig. 1) : on peut en conclure que l'adsorption de l'atrazine est d'autant plus grande que la charge négative en surface est plus grande. Dans la zone de $\mathrm{pH}$ étudiée, la molécule d'atrazine ne se protone pas en solution. Cependant, lors de la mesure d'adsorption, l'apport d'une solution aqueuse d'atrazine à $\mathrm{pH}$ 6,6 va provoquer la dissociation des $\mathrm{OH}$ superficiels des surfaces latérales de la kaolinite et la libération de protons dans la solution, dont un certain nombre va se trouver en plus forte concentration dans la partie diffuse de la double couche ionique entourant les cristallites d'argile. La molécule d'atrazine étant très polaire $\left(\mu=4,63 \times 10^{18}\right.$ u.e.s $)$, elle ira au voisinage de la surface où le $\mathrm{pH}$ sera plus bas que dans la solution et là, elle sera susceptible de se protoner. L'adsorption varie dans le sens kaolinite $\mathrm{Na}>$ kaolinite $\mathrm{Ca}>$ kaolinite $\mathrm{Al}$ alors que l'affinité des cations pour la surface chargée négativement varie dans le sens $\mathrm{Al}>\mathrm{Ca}>\mathrm{Na}$. Ces résultats incitent à dire que le mécanisme de fixation, même en milieu neutre, est principalement dû à une protonation de la molé iule organique (à caractère base faible) dans la partie diffuse de la double couche au voisinage de la surface, puis à des interactions coulombiennes entre la molécule chargée négativement.

Ces hypothèses sont de plus confortées par les travaux de BAILEY \& KARICKHOFF (1973), de KARICKHOFF \& BAILEY (1976), de FELDKAMP \& WhITE (1979 a et b) et de FELDKAMP et al. (1981). Ces auteurs montrent, à l'aide d'expériences effectuées en spectroscopie d'adsorption 
ultra-violette, que les surfaces des argiles, même dans des suspensions très diluées, sont suffisamment acides pour déplacer la réaction acido-basique des triazines et que l'intensité de la protonation des molécules ne dépend pas du $\mathrm{pk}_{\mathrm{BH}}+$ de ces bases faibles, mais plutôt de l'affinité de la molécule organique protonée pour l'interface eau-argile.

\section{B. Adsorption de l'atrazine par la montmorillonite}

Contrairement à la kaolinite, la montmorillonite possède une charge négative permanente très importante due aux substitutions isomorphiques du réseau (de l'ordre de
$90 \mathrm{meq} / 100 \mathrm{~g}$ ) et une charge variable très faible due à la dissociation des groupements hydroxylés des surfaces latérales (de l'ordre de quelques meq/100 g), comme dans le cas de la kaolinite (VAN OLPHEN, 1977; GREENLAND \& HAYES, 1978). La comparaison entre la montmorillonite et la kaolinite est de ce fait difficile. Ainsi, la simple mesure de la variation du $\mathrm{pH}$ des solutions après contact avec la montmorillonite Na (tabl. 3) n'est pas suffisante pour expliquer la différence de comportement des 2 argiles vis-à-vis des protons. On peut cependant attribuer la disparition des protons de la solution à un échange ionique entre $\mathrm{Na}^{+}$et $\mathrm{H}^{+}$, la quantité de $\mathrm{H}^{+}$fixé par $\mathrm{g}$ de montmorillonite étant d'autant plus grande que la suspension est diluée.

TABLEAU 3

Influence de la dilution de la montmorillonite $\mathrm{Na}$ dans une suspension aqueuse, sur la fixation de protons à partir de la solution. Influence of the $\mathrm{Na}$ montmorillonite dilution in an aqueous suspension on proton fixation from the solution.

\begin{tabular}{|c|c|c|c|c|}
\hline $\mathrm{pH}$ initial & $\begin{array}{l}\text { Concentration } \\
\text { des suspensions } \\
\text { de montmorillonite }\end{array}$ & $\begin{array}{l}\mathrm{pH} \text { final } \\
\text { de la } \\
\text { solution }\end{array}$ & $\begin{array}{c}{\left[\mathrm{H}^{+}\right] \text {disparue }} \\
\text { de la } \\
\text { solution }\end{array}$ & $\begin{array}{c}\text { Fixation } \\
\text { de }\left[\mathrm{H}^{+}\right] / \mathrm{g} \\
\text { de montmorillonite }\end{array}$ \\
\hline $6,93 \pm 0,01$ & $\begin{array}{cc}2,5 & \mathrm{~g} / 1 \\
5 & \mathrm{~g} / 1 \\
10 & \mathrm{~g} / 1\end{array}$ & $\begin{array}{l}7,20 \pm 0,01 \\
7,28 \pm 0,01 \\
7,38 \pm 0,01\end{array}$ & $\begin{array}{l}0,63 \times 10^{-7} \\
0,52 \times 10^{-7} \\
0,42 \times 10^{-7}\end{array}$ & $\begin{array}{r}21,6 \times 10^{-9} \\
13,0 \times 10^{-9} \\
7,5 \times 10^{-9}\end{array}$ \\
\hline
\end{tabular}

Des résultats expérimentaux récents (M. TERCE, résultats non publiés, 1982) montrent que la présence de particules de montmorillonite augmente considérablement la constante de dissociation $\left(\mathrm{pk}_{\mathrm{BH}}+\right)$ de l'équilibre acidobasique de l'atrazine. Le $\mathrm{pK}_{\mathrm{BH}}+$ de l'atrazine dans l'eau pure est de 1,68. Les $\mathrm{pK}_{\mathrm{BH}}+$ apparents déterminés en présence de particules de montmorillonite diffèrent selon la nature du cation saturant l'argile : ils sont respectivement de 4,3 pour le sodium, de 3,3 pour le calcium et de 2,7 pour l'aluminium.

On peut supposer que le mécanisme de fixation de l'atrazine par la montmorillonite est le même que celui par la kaolinite:

- Attraction des molécules polaires d'atrazine par des surfaces chargées négativement.

- Protonation des molécules au voisinage des surfaces où la concentration en protons est importante.

- Interaction entre la molécule protonće et la surface chargée négativement, qui devrait être facilitée dans le sens $\mathrm{Na}>\mathrm{Ca}>\mathrm{Al}$.

Les résultats expérimentaux (fig. 3b) montrent cependant que l'adsorption est plus importante quand l'aluminium cst le cation échangeable. Ce phénomène peut s'expliquer de la façon suivante: l'ion $\mathrm{Al}\left(\mathrm{H}_{2} \mathrm{O}\right)_{6}{ }^{3+}$ à la surface de la montmorillonite a un caractère acide faible et peut se trouver dissocié, selon les conditions de $\mathrm{pH}$, soit sous des formes échangeables $\left[\begin{array}{lll}\mathrm{Al} & \left(\mathrm{H}_{2} \mathrm{O}\right)_{5} & \mathrm{OH}\end{array}\right]^{2+}, \quad\left[\begin{array}{lll}\mathrm{Al} & \left(\mathrm{H}_{2} \mathrm{O}\right)_{4}\end{array}\right.$ $\left.(\mathrm{OH})_{2}\right]^{+}$, soit éventuellement sous des formes polymérisées non échangeables, avec libération de protons situés au voisinage de la surface. Ainsi, selon JACKSON (1963), le cation trivalent serait dissocié à 50 p. 100 à pH 5. Or, le pH des suspensions de montmorillonite $\mathrm{Al}$ utilisées pour faire des mesures est de l'ordre de 5,5 et la dissociation partielle des ions $\mathrm{Al}\left(\mathrm{H}_{2} \mathrm{O}\right)_{6}{ }^{3+}$ augmente lá présence de protons au niveau de l'interface, donc intensifie la protonation des molécules d'atrazine et, par conséquent, les quantités fixées.
L'origine de la variation de l'acidité de surface de la montmorillonite est actuellement difficile à définir. Le fait que l'adsorption par la montmorillonite $\mathrm{Na} \mathrm{(ou} \mathrm{Ca)} \mathrm{est}$ sensiblement du même ordre de grandeur que l'adsorption par la kaolinite $\mathrm{Na}$ (ou $\mathrm{Ca}$ ) permet simplement de dire qu'en milieu neutre et en suspension diluée, les surfaces des argiles $\mathrm{Na}$ (ou $\mathrm{Ca}$ ) ont des aptitudes très voisines à protoner les molécules d'atrazine.

\section{CONCLUSION}

L'atrazine, molécule à caractère acido-basique, semble être un bon indicateur de l'acidité de surface de la montmorillonite ou de la kaolinite, même dans des suspensions très diluées. L'acidité de surface déplace la réaction acide-base et une base très faible telle que l'atrazine peut se trouver sous une forme protonée au voisinage de la surface, alors que le $\mathrm{pH}$ de la suspension aqueuse ne le laisserait pas supposer.

Plus les suspensions des 2 argiles sont diluées, plus l'aptitude de l'interface eau-argile à protoner la base faible organique est grande et, par conséquent, plus l'adsorption d'atrazine par gramme d'adsorbant est importante.

Si l'explication de l'origine de la variation de l'acidité de surface de la kaolinite est relativement aisée, elle l'est beaucoup moins avec la montmorillonite sodique ou calcique. En suspension diluée, il n'a jamais été montré que l'eau d'hydratation des cations $\mathrm{Na}$ ou $\mathrm{Ca}$ ont des propriétés acides (MORTLAND, 1968).

Dans le cas de la montmorillonite $\mathrm{Al}$, la présence d'ions Al $\left(\mathrm{H}_{2} \mathrm{O}\right)_{6}{ }^{3+}$ à caractère acide faible augmente le nombre de protons au niveau de l'interface, donc l'acidité de surface.

Ces résultats montrent que la diversité des résultats publiés relatifs à l'adsorption de l'atrazine (tabl. 1) est due à une modification des propriétés acides des interfaces argiles-eau, selon la dilution de l'argile. 
Enfin, l'effet de dilution de l'adsorbant sur la fixation de l'atrazine montre qu'il est vain, dans un but comparatif, de vouloir exprimer les quantités de produit fixé par masse d'adsorbant ou par aire de surface. L'adsorption doit être directement reliée à une densité de charge négative varia- ble, tout au moins dans le cas de la kaolinite, celui de la montmorillonite étant encore beaucoup trop complexe.

Reçu le 22 juin 1982. Accepté le 11 mai 1983.

\section{RÉFÉRENCES BIBLIOGRAPHIQUES}

Bailey G. W., Karickhoff S. W., 1973. An ultraviolet spectroscopic method for monitoring surface acidity of clay minerals under varying water content. Clays clay Miner., 21, 471-477.

Bailey G. W., White J. L., Rothberg T., 1968. Adsorption of organic herbicides by montmorillonite. Role of $\mathrm{pH}$ and chemical character of adsorbate. Soil Sci. Soc. Am. Proc., 32, 222-234.

Bar-on P., Shainberg I., 1970. Hydrolysis and decomposition of Namontmorillonite in distilled water. Soil Sci., 109, 4, 241-246.

Cases J. M., 1968. Les phénomènes physico-chimiques à l'interface. Application au procédé de la flottation. Sciences de la terre, mémoire $\mathrm{n}^{\circ} 13,120 \mathrm{p}$.

Cases J. M., 1979. Adsorption des tensio-actifs à l'interface solideliquide : thermodynamique et influence de l'hétérogénéité des adsorbants. Bull. Minér., 102, 684-707.

Cases J. M., Touret-Poinsignon C., Vestier D., 1971. Etude des phénomènes électrochimiques par titration potentiométrique et électrophorèse : cas de la kaolinite. C. R. Acad. Sic., Paris, Séric C, 272, 728-731.

Ekka E., Fripiat J. J., 1957. Etude des groupes hydroxyles de surface de la kaolinite. II. Variation de la capacité d'échange de bases de la kaolinite en fonction du pH. Pédologie, VII. Gand. 3950.

Feldkamp J. R., White J. L., 1979. a) Acid-base equilibria in clay suspension. J. Colloid. Interface Sci., 69, 1, 97-106.

Feldkamp J. R., White J. L., 1979. b) Protonation of bases in clay suspensions. Dev. Sedimentol., 27, 187-196.

Feldkamp J. R., White J. L., Browne J. E., Hem S. L., 1981. Displacement of acid-base equilibria in clay suspensions as calculated from double layer theory. J. Colloild. Interface. Sci., 80, 1, 6773.

Frenkel H., Suarez D. L., 1977. Hydrolysis and decomposition of calcium montmorillonite. Soil Sci. Soc. Am. J., 41, 5, 881-891.

Fripiat J. J., 1957. Propriétés de surface des alumino-silicates. Bull. Groupe fr. Argiles, IX, 23-47.

Greenland D. J., Hayes M. H. B., 1978. The chemistry of soil constituents. Ed. by Greenland D. J. and Hayes M. H. B. Chichester: John Wiley and Sons. A Wilev. Interscience publication p. 322-326.
Jackson M. L., 1963. Aluminium bonding in soils: a unifying principle in Soil Science. Soil Sci. Soc. Amer. Proc., 27, 1, 1-10.

Karickhoff S. W., Bailey G. W., 1976. Protonation of organic bases in clay-water systems. Clays Clay Miner., 24, 170-176.

Mortland M. M., 1968. Protonation of compounds at clay mineral surfaces. 9 th int. Congr. Soil Sci., 1, 691-699.

Pons C. H., 1980. Mise en évidence des relations entre la texture et la structure dans "les systèmes eau-smectites par diffusion aux petits angles de rayonnement $X$ synchrotron. Thèse présentée à l'Université d'Orléans. UER de Sciences fondamentales et appliquées, $175 \mathrm{p}$.

Schofield R. K., Samson H. R., 1953. The deflocculation of kaolinite suspensions and the accompanying change-over from positive to negative chloride adsorption. Clay Miner. Bull., 2, 45-51.

Terce M., Calvet R., 1974. Adsorption de l'atrazine sur des argiles bi-ioniques Ca-Al. C. R. Acad. Sci. Paris., 279, 1859-1862.

Terce M., Clavet R., 1975. Rôle de l'aluminium dans l'adsorption de l'atrazinc. Proc. Symp. Israël. France. Behaviour of pesticides in soil, 33-39.

Terce M., Calvet R., 1977. Some observations on the role of Al and $\mathrm{Fe}$ and their hydroxides in the adsorption of herbicides by montmorillonite. Proc. Symp. Herbicides-Soil. Stuttgart, 237-243. Vallet M., Calvet R., Chaussidon J., 1973. Remarques complémentaires sur quelques aspects physico-chimiques de l'adsorption de l'atrazine par les montmorillonites. Proc. Eur. Weed Res. Counc. Symp. Herbicides-Soil., Versailles, 41-50.

Van Olphen H., 1977. An introduction to clay colloid chemistry, p. 92-95. Second edition A Wiley-Interscience publication. New-York $318 \mathrm{p}$.

Vestier D., 1969. Evolution du potentiel électrocinétique de la kaolinite en milieu aqueux. Sciences de la terre, XIV, 4, 292-300. Weber J. B., 1970. Adsorption of s-triazines by montmorillonites as a function of $\mathrm{pH}$ and molecular structure. Soil Sci. Soc. Am. Proc., 34, 3, 401-404. 